\title{
Special Issue on Flexible Molecular Descriptors
}

\author{
Andrey A. Toropov ${ }^{1}$ and Eduardo A. Castro ${ }^{2, *}$ \\ ${ }^{1}$ Vostok Holding Innovation Company, Sadik Azimov 4th Street, 15, Tashkent 700000, Uzbekistan \\ ${ }^{2}$ INIFTA, Suc.4, C.C. 16, La Plata 1900, Argentina \\ * Author to whom correspondence should be addressed; e-mail: castro@quimica.unlp.edu.ar
}

Received: 1 November 2004 / Accepted: 2 November 2004 / Published: 31 December 2004

Molecular indices are characterized by fixed numerical values, which are independent of the physical chemistry property or/and biological activity considered. Once the bonding pattern of a molecule is defined they can then be computed. Contrarily, variable indices depend on the property/activity under consideration. Thus, variable descriptors are functions instead of numerical data and they change during the regression analysis. This more general definition of molecular indices has offered a powerful tool for studies within the realm of QSAR/QSPR theory and it has enabled authors to surmount the so called "nightmare of molecular descriptors". This drawback arises when one has to choose among a huge number of possibilities to assign a set of molecular descriptors to perform a given study. In fact, the optimization of molecular descriptors for the particular application makes it unnecessary to focus the attention on the choice of a particular set of indices and the efforts can then be directed towards the suitable search of the best mathematical algorithm to obtain the corresponding flexible descriptors.

Flexible (optimal) descriptors enable one to define molecular fragments, which are increasing (reducing) numerical values of the modeled physical or chemical or biological parameter. Thus, flexible descriptors often enable formulation of mechanistic interpretations of models arising from their use. Despite this rather attractive feature of flexible molecular descriptors, their use is not quite as extended as one would assume, on the basis of the previous comments. This special issue of Molecules devoted to flexible molecular descriptors tries to surmount this deficiency through the valuable contributions of many well recognized authors on this field. The Guest Editors thank them warmly for their kind collaboration.

Andrey A. Toropov and Eduardo A. Castro

C 2004 by MDPI (http://www.mdpi.org). Reproduction is permitted for noncommercial purposes. 